University of Nebraska - Lincoln

DigitalCommons@University of Nebraska - Lincoln

\title{
A Heuristic with Bounded Guarantee to Compute Diverse Paths under Shared Protection in WDM Mesh Networks
}

Ajay Todimala

University of Nebraska-Lincoln, ajayt@cse.unl.edu

Byrav Ramamurthy

University of Nebraska-Lincoln, bramamurthy2@unl.edu

Follow this and additional works at: https://digitalcommons.unl.edu/cseconfwork

Part of the Computer Sciences Commons

Todimala, Ajay and Ramamurthy, Byrav, "A Heuristic with Bounded Guarantee to Compute Diverse Paths under Shared Protection in WDM Mesh Networks" (2005). CSE Conference and Workshop Papers. 97. https://digitalcommons.unl.edu/cseconfwork/97

This Article is brought to you for free and open access by the Computer Science and Engineering, Department of at DigitalCommons@University of Nebraska - Lincoln. It has been accepted for inclusion in CSE Conference and Workshop Papers by an authorized administrator of DigitalCommons@University of Nebraska - Lincoln. 


\title{
A Heuristic with Bounded Guarantee to Compute Diverse Paths under Shared Protection in WDM Mesh Networks
}

\author{
Ajay Todimala and Byrav Ramamurthy \\ Department of Computer Science and Engineering \\ University of Nebraska-Lincoln \\ Lincoln NE 68588-0115 U.S.A. \\ Email: \{ajayt, byrav\}@cse.unl.edu
}

\begin{abstract}
Establishing a fault-tolerant connection in a network involves computation of diverse working and protection paths. The Shared Risk Link Group (SRLG) [1] concept is used to model several types of failure conditions such as link, node, fiber conduit, etc. In this work we focus on the problem of computing optimal SRLG/link diverse paths under shared protection. Shared protection technique improves network resource utilization by allowing protection paths of multiple connections to share resources. In this work we propose an iterative heuristic for computing SRLG/link diverse paths. We present a method to calculate a quantitative measure that provides a bounded guarantee on the optimality of the diverse paths computed by the heuristic. The experimental results on computing link diverse paths show that our proposed heuristic is efficient in terms of number of iterations required (time taken) to compute diverse paths when compared to other previously proposed heuristics.
\end{abstract}

\section{INTRODUCTION}

Wavelength division multiplexing (WDM) technique allows connections to be multiplexed onto a single fiber link, each on a different wavelength. An end-to-end connection spanning multiple fiber links is established by assigning a wavelength along each fiber link. The optical switches at intermediate nodes switch an optical wavelength signal from an input port to an output port, without any opto-electronic conversions. The end-to-end optical light path connection with no optoelectronic conversions at the intermediate nodes is called a lightpath. With no wavelength conversion capability at intermediate optical switches a connection must be assigned same the wavelength along all the fibers from the source to the destination.

The physical topology of a WDM network consists of nodes interconnected with one or more pairs of fiber links. The fiber links are stuffed in conduits which are laid along right-of-way such as a railway track. A conduit may consist of fiber links of more than one pair of nodes. When a failure occurs in a conduit such as a conduit cut, all the fiber links in the conduit will fail at the same time resulting in failure of multiple physical links (optical fiber links).

The concept of Shared Risk Link Group (SRLG), a formal model to model failures in an optical network was introduced in [1]. An SRLG is any sub-set of links in the network that share the risk of failing at the same time. SRLG can be used to model several types of failure conditions such as single-link failures, node failures, conduit failures, double-link failures or failure of any other possible subset of links sharing a common risk. Such an SRLG is called a general SRLG. In this work although we present a generic heuristic to handle SRLG failures we only present the experimental results for single link failures in the network.

To establish a fault-tolerant connection in a network we need to compute two diverse paths, usually called working and protection paths, such that both the paths do not fail at the same time when failures occur in the network. For a connection to be fault tolerant against SRLG failures in the network the working and protection paths must be SRLG diverse. A fault tolerant connection can be protected in two ways: dedicated protection and shared protection. In dedicated protection, a connection is established on working as well as protection paths and the data is sent on both the paths, similar to establishing two connections. Shared protection allows protection paths of different connections to share resources when their corresponding working paths do not share the risk of failing at the same time i.e., if the working paths do not share fiber links or SRLG, referred as backup multiplexing.

In this work we propose an iterative heuristic to compute optimal SRLG/link-diverse paths under shared protection. We present a method to compute a quantitative measure that provides a bounded guarantee on the optimality of the sub-optimal solution computed by our proposed iterative heuristic. The rest of the paper is organized as follows. In Section II we discuss related work on computing diverse paths. In Section III 
we present our iterative modified network-flow heuristic (IMNH) with its proof of correctness. In Section IV we discuss the experimental results and present concluding remarks in Section V.

\section{RElAted Work AND Motivation}

In this section, we first briefly survey the related work on computing link-diverse path pair problem under dedicated and shared protection. We then present related research on the problem of computing a SRLG-diverse path pair.

The two-step heuristic (TSH) is a simple heuristic to compute a pair of edge disjoint paths between a pair of nodes in a graph $G$. TSH sometimes fails to compute link-diverse paths, even though they exist, the so-called trap topology problem. Most of the research studies on computing diverse paths for shared protection assume that the cost of using a link for a protection path is only a fraction of the cost of using the link for a working path. Such paths are said to be asymmetrically weighted [2] paths. In shared protection the optimal path pair is defined as the asymmetrically-weighted least-cost link-diverse path pair (AL-LDP). The AL-LDP problem is studied in [2], [3], and [4]. While most of the literature focussed on disjoint paths without considering wavelength continuity constraint, the authors in [5] proved that the problem of computing disjoint path pair with wavelength continuity constraint is NP-complete.

Computing SRLG-diverse paths problem is proved to be NP-complete [6]. The trap topology problem is more prominent when considering SRLG diversity than link diversity. Most of the research on computing SRLGdiverse paths focuses on countering the trap topology problem but does not consider the optimality of the path pair. The optimality of the path pair computed depends upon the protection technique being used, dedicated or shared. Many heuristics have been proposed to compute SRLG diverse paths. The paper [7] proposes the BasicLink method and the Bypass method to compute SRLG-diverse paths. In [8] an ILP based approach is used to solve the SRLG diverse routing problem in a network with static traffic. The paper [9] proposes a trap avoidance algorithm for computing SRLG-diverse paths that uses an iterative approach. In [10] the working path is partitioned into overlapping segments and each individual segment is protected using a backup path. In paper [11] a graph based approach is used to present an algorithm for computing SRLG-diverse paths, but it only works for SRLGs consisting of links incident on a single node. In [12] an iterative heuristic for computing SRLG-diverse paths for dedicated protection is proposed. Most of these existing heuristics only consider minimizing the sum of the costs of the SRLGdiverse paths i.e., computing SRLG-diverse paths for dedicated protection.

In this work we focus on the problem of computing optimal SRLG/link diverse paths under shared protection. We consider the definition of an optimal SRLG diverse paths under shared protection as asymmetrically-weighted least cost SRLG-diverse paths (AL-SDP). The Iterative two-step heuristic (ITSH) [13] is one of the few better known heuristics for solving the AL-SDP problem under shared protection. In the next section we present an iterative heuristic as an improvement to ITSH. Our proposed heuristic is faster than ITSH in computing and verifying the optimality of the diverse path pair. As AL-SDP problem is NP-complete [6], our iterative heuristic, similar to ITSH, does not guarantee the computation of the optimal solution within a specified number of iterations (polynomial time).

\section{OUR Heuristics}

In this section we present our iterative modified network-flow heuristic (IMNH) for solving both AL-SDP and AL-LDP problems. The IMNH heuristic uses the modified network-flow algorithm for SRLG Diversity (MNA-SD) and modified network-flow algorithm (MNA) in solving for AL-SDP and ALLDP problems respectively. In this section we first present the IMNH heuristic and then present the MNASD algorithm. We discuss the MNA algorithm while discussing MNA-SD later in this section.

\section{A. Iterative Modified Network-flow Heuristic (IMNH)}

The IMNH heuristic is outlined in Algorithm 1. The input to the IMNH heuristic is a graph $G=(V, E)$, its edge-cost function $C$, the asymmetric weight $w, w \geq 1$, and a node pair $(s, t)$. The IMHN heuristic outputs the best path pair computed within the specified time limit (iteration limit). In Steps $1-2$, IMNH initializes the current best diverse pair and its cost to $N U L L$ and $\infty$ respectively. The IMNH heuristic iterates steps 4 to 14 until the number of iterations exceed $M$, a predetermined iteration limit. The IMNH heuristic starts each iteration by computing the $i^{\text {th }}$ shortest path from $s$ to $t$ in step 5 . The $i^{t h}$ shortest path is computed using a $K$-shortest path algorithm such as Yen's algorithm [14]. The IMNH heuristic using the $i^{t h}$ shortest path as the input seed path invokes the MNA-SD algorithm to compute the SRLG-diverse path pair. If MNASD successfully computes an SRLG-diverse path pair $\left(p_{i}^{\prime}, p_{i}^{\prime \prime}\right)$ and its cost is less than the cost of current best diverse pair found so far, IMNH updates the current best 
diverse pair in Steps 8 - 9. If SRLG-diverse path pair could not be computed in an iteration, the procedure continues with the next iteration. Like ITSH, IMNH continues to iterate until an optimal solution is found or until the pre-determined iteration limit $M$ is reached.

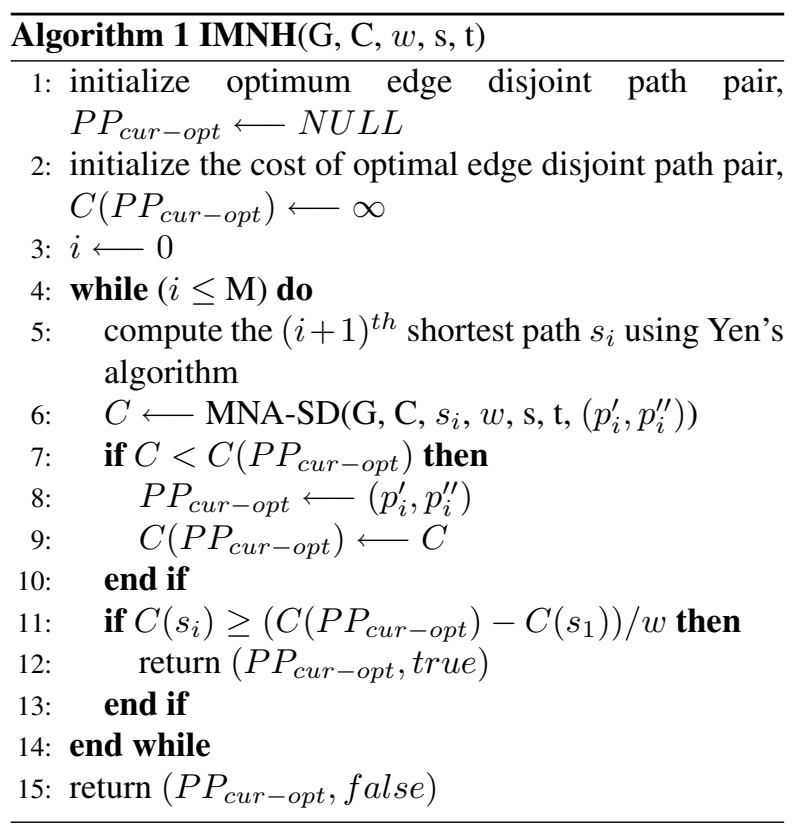

1) Optimality Verification and Measure: In this section we state and prove Theorems 1 and 2 which are used by the optimality verification criterion of the IMNH heuristic and to quantitatively measure the optimality of the solution computed by IMNH respectively. Theorems 1 states that when the cost of the seed path reaches or exceeds $C\left(P P_{\text {cur-opt }}\right)-\left(w \times C\left(s_{1}\right)\right)$, the current best path pair $P P_{\text {cur-opt }}$ is the optimal solution. Therefore IMNH stops and outputs the path pair $P P_{\text {cur-opt }}$ as the optimal solution. A close observation will reveal that using a similar argument the optimality verification of the ITSH heuristic can also be enhanced so that ITSH stops when seed path cost exceeds $C\left(P P_{\text {cur-opt }}\right) /(w+$ 1) instead of $C_{\text {cur-opt }}$.

If the optimal path pair could not be computed by IMNH even after $M$ iterations, we can compute a quantitative measure of the optimality of the suboptimal solution computed using the result of Theorem 2. Theorem 2 states that after completing $i$ iterations if the optimality could not be verified then the cost of the current best path pair $P P_{\text {cur-opt }}, C_{c u r-o p t}$, is at most $\frac{C_{c u r-o p t}}{\left(w C\left(s_{1}\right)+C\left(s_{i}\right)\right)}$ times the cost of the optimal solution. Therefore, at the end of $M$ iterations if the optimal solution is not found, the fraction $\frac{C_{c u r-o p t}}{\left(w C\left(s_{1}\right)+C\left(s_{i}\right)\right)}$ gives at most how far optimal is the path pair $P P_{\text {cur-opt }}$ from the optimal path pair. Thus providing a bounded guarantee on the optimality of the path pair $P P_{\text {cur-opt }}$.
Theorem 1. Let $P P_{\text {cur-opt }}$ be the current optimal disjoint path pair computed by IMNH algorithm at the end of $i^{\text {th }}$ iteration, $C_{\text {cur-opt }}$ be its weighted cost and let $C\left(s_{i}\right)$ be the cost of the $i^{\text {th }}$ shortest path in graph $G$ then if $C\left(s_{i}\right) \geq C_{\text {cur-opt }}-\left(w \times C\left(s_{1}\right)\right)$ then $P P_{\text {cur-opt }}$ is the optimal disjoint path pair.

Proof: Suppose $P P_{\text {cur-opt }}$ is not optimal and let $(p, q)$ the disjoint path pair computed in $j^{\text {th }}$ iteration, $(j>i)$, of IMNH algorithm is optimal and $C(p) \leq$ $C(q)$. Then,

$$
C(p) \times w+C(q)<C_{\text {cur-opt }}
$$

The IMNH heuristic considers the seed paths for each iteration in the non-decreasing order of costs. If the cost of paths $s_{i}$ and $s_{j}$ in the $i^{t h}$ and $j^{t h}$ iteration respectively are $C\left(s_{i}\right)$ and $C\left(s_{j}\right)$ and since $i<j$, then $C\left(s_{i}\right) \leq$ $C\left(s_{j}\right)$. From the MNA algorithm, we know that at least one of the paths $p, q$ has cost at least $C\left(s_{j}\right)$. Without loss of generality, let $C\left(s_{j}\right) \leq C(q)$. Therefore $C\left(s_{i}\right) \leq$ $C(q)$. And also $C\left(s_{1}\right) \leq C(p)$ where $C\left(s_{1}\right)$ is the cost of the shortest path. From Eq. 1 we get,

$$
\begin{aligned}
w \times C\left(s_{1}\right)+C\left(s_{i}\right) & <C_{\text {cur }-o p t} \\
C\left(s_{i}\right) & <C_{\text {cur }-o p t}-\left(w \times C\left(s_{1}\right)\right),
\end{aligned}
$$

a contradiction.

Theorem 2. Let PP $P_{\text {cur-opt }}$ be the current optimal disjoint path pair computed by IMNH algorithm at the end of $i^{\text {th }}$ iteration, $C_{\text {cur-opt }}$ be its weighted cost, let $C\left(s_{i}\right)$ be the cost of the $i^{\text {th }}$ shortest path in graph $G$ and optimal not found then $C_{\text {cur-opt }}$ is at most $\frac{C_{\text {cur-opt }}}{\left(w C\left(s_{1}\right)+C\left(s_{i}\right)\right)}$ times optimal solution.

Proof: Let opt be cost of the optimal disjoint path pair $(p, q)$ i.e., opt $=w p+q$. Now, opt $\geq w C\left(s_{i}\right)+$ $C\left(s_{1}\right)$, since if opt $<w C\left(s_{1}\right)+C\left(s_{i}\right)$ the optimal path pair $(p, q)$ must have already been computed in some iteration $j, j<i$ where $C\left(s_{j}\right)<C\left(s_{i}\right)$, a contradiction. Now consider the ratio, $\frac{C_{c u r-o p t}}{o p t} \leq \frac{C_{c u r-o p t}}{\left(w C\left(s_{1}\right)+C\left(s_{i}\right)\right)}$. Hence proved.

\section{B. Modified Network-flow Algorithm for SRLG Diversity (MNA-SD)}

In this section, we present the modified network-flow algorithm for computing SRLG-diverse paths, outlined in Algorithm 2. The input to the MNA-SD algorithm is same as that of IMNH and an additional seed path $p$. The algorithm computes a pair of SRLG-diverse paths, if a diverse path pair can be computed by avoiding trap SRLG links along the seed path $p$, otherwise it returns NULL. Trap SRLG links along a path $p$ are defined as the links along path $p$ that are responsible for the 


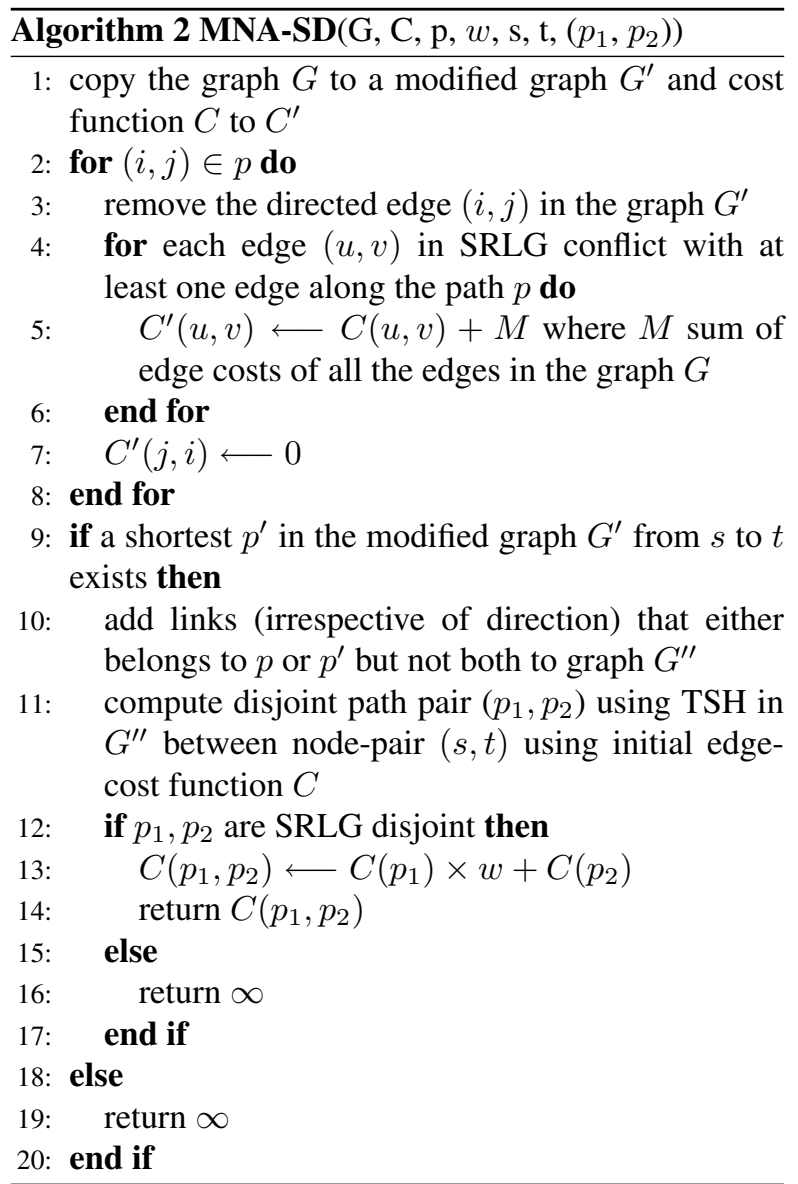

failure of TSH in computing disjoint path pair. MNASD identifies trap SRLG links along the given path (seed path). In Steps 1 - 10 MNA-SD identifies and removes the trap SRLG links along the seed path $p$. In Steps 10 it adds all the links along the paths $p$ and $p^{\prime}$ except the trap SRLG links to a temporary graph $G^{\prime \prime}$. In Steps $4-7$ all the links that are in SRLG conflict with at least one link along the seed path $p$ are assigned a cost of $M$ where $M$ is the sum of costs of all the links in the graph $G$. This is done to avoid using links that are in SRLG conflict with the path $p$ while computing path $p^{\prime}$ in Step 9. In step 11, the MNA-SD algorithm computes paths $p_{1}$ and $p_{2}$ using only links that exclusively belong to either path $p$ or $q$ but not both. Let $p_{1}$ be the path whose cost is at least the cost of the seed path $p$. If the costs of both the paths $p_{1}$ and $p_{2}$ are greater than the cost of the seed path $p$ or if costs of both the paths $p_{1}$ and $p_{2}$ are equal then let $p_{1}$ be the path that has more links in common with the seed path $p$ in comparison to path $p_{2}$. In step 12, MNA-SD checks to see that the link-diverse paths are also SRLGdiverse. If $\left(p_{1}, p_{2}\right)$ are SRLG-diverse MNA-SD returns $\left(p_{1}, p_{2}\right)$ and its sum of asymmetrically weighted costs of $\left(p_{1}\right.$ and $\left.p_{2}\right)$. Otherwise MNA-SD fails and returns $\infty$.
For link diversity, IMNH uses MNA. MNA-SD can be easily modified to obtain MNA. To obtain MNA remove the Steps $4-7$ in MNA-SD and return the path pair $\left(p_{1}, p_{2}\right)$ computed in Step 11. MNA identifies the trap links along the given input seed path and computes link-diverse paths. MNA therefore can avoid trap topology problem.

1) Complexity: Let us analyze the complexity of the MNA-SD. Steps $2-9$ take $O(n)$ time, assuming that the seed path has no cycles and the number of edge in an SRLG is at most at constant $k$. Since the edges in the modified graph $G^{\prime}$ have non-negative costs, the graph $G^{\prime}$ has no negative cycles. Therefore Dijkstra's algorithm can be used to compute the shortest path in Step 10. We know that the time complexity of Dijkstra's algorithm is $O(m+n \log n)$ where $n$ is the number of nodes and $m$ is the number of links in the network. Steps 10 can be computed in $O\left(n^{2}\right)$. In Step 11 the TSH can use Dijkstra's algorithm to compute the diverse paths in $G^{\prime \prime}$. Using TSH to compute diverse paths in Step 11, the complexity is $O(m+n \log n)$. Therefore the time complexity of the MNA-SD is $O\left(m+n \log n+n^{2}\right)$.

A close observation at the working of the algorithm will reveal that the paths computed in Step 11 use only the links along the seed path $p$ and path $p^{\prime}$ but not the links belonging to both the paths. From this observation Step 11 can be modified to compute the diverse paths by a simple rearrangement in $O(n)$ time. With this enhancement, like TSH, MNA-SD invokes Dijkstra's algorithm only once. Hence the running times of TSH and MNA-SD can be considered equivalent.

\section{ExPerimental Design and Results}

In this paper we only present the results for the case of link diversity shown in Fig. 1 and 2. We conducted experiments on a 79-node network with 108 links and 20 wavelengths, used in other works such as [3]. We simulated dynamic traffic with calls arriving into the network having Poisson distribution with mean arrival rate $\lambda$. The connections have exponential holding time with mean $1 / \mu$. The load on the network is measured as $\lambda / \mu$. The number of calls simulated in the network during each simulation run are 1,000,000. We implemented the ITSH, IMNH algorithms. The ITSH and IMNH heuristics with iteration limit of $k$ and asymmetric weight $w$ are referred to as ITSH-kP-Ww and IMNH-kP-Ww respectively. The performance is measured in terms of blocking probability. The blocking probability is the ratio of the number of blocked calls to the total number of calls simulated. The experiments are run on a lightly loaded Sun Fire V210 with 2 UltraSPARC IIIi $1 \mathrm{GHz}$ processors and 2 GB RAM. 


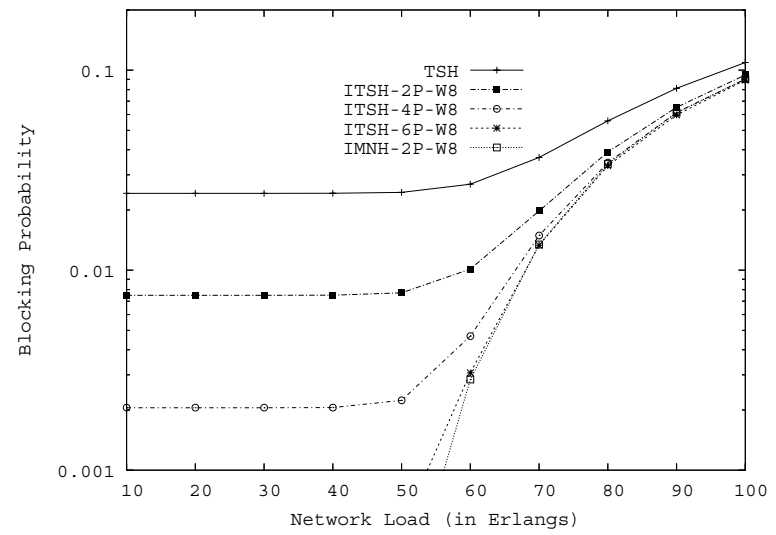

Fig. 1. The blocking probability verses load (in Erlangs) of simulating $1,000,000$ calls on 79-node network with no wavelength conversion for heuristics TSH, ITSH with iteration limit of 2, 4, 6 and IMNH with iteration limit of 2 and asymmetric weight 8 .

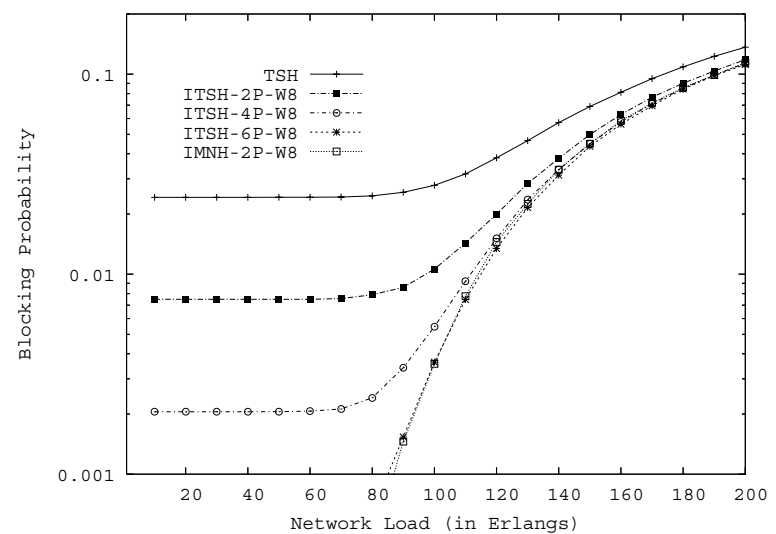

Fig. 2. The blocking probability verses load (in Erlangs) of simulating $1,000,000$ calls on 79-node network with full wavelength conversion for heuristics TSH, ITSH with iteration limit of 2, 4, 6 and IMNH with iteration limit of 2 and asymmetric weight 8 .

Figures 1 and 2 plot the blocking probability with increasing network load in Erlangs for heuristics TSH, ITSH and our proposed IMHN heuristic on the 79node network with no and full wavelength conversion respectively. The performance of the ITSH when the iteration limit is 6 is equivalent to that of our IMNH heuristic with iteration limit of 2 .

\section{CONCLUSIONS}

In this work we studied the problem of computing optimal SRLG/link diverse paths under shared protection in optical WDM networks. We consider the definition of an optimal SRLG diverse paths under shared protection as asymmetrically-weighted least cost SRLG-diverse paths (AL-SDP) (correspondingly, AL-LDP for link diverse paths). We propose a generic iterative modified network-flow heuristic (IMNH) to solve both AL-SDP and AL-LDP problems. IMNH uses
MNA-SD and MNA for solving AL-SDP and AL-LDP problems respectively. We presented both MNA-SD and MNA algorithms. We also presented a method to compute a quantitative measure that provides a bounded guarantee on the optimality of the solution computed by our IMNH heuristic. Our experiments on a 79-node network showed that the blocking performance of our proposed IMNH heuristic with an iteration limit of 2 is equivalent to the blocking performance of ITSH with an iteration limit of 6 .

\section{REFERENCES}

[1] J. Strand, A. L. Chiu, and R. Tkach, "Issues for Routing in the Optical Layer," IEEE Communications Magazine vol. 39, issue. 2, pp. 81-87, Feb 2001.

[2] P. Laborczi, et. al., "Solving asymmetrically weighted optimal or near-optimal disjoint path pair for the survivable optical networks," in Third International Workshop On Design Of Reliable Communication Networks (DRCN '01), Oct. 2001.

[3] D. Xu, Y. Chen, Y. Xiong, C. Qiao, and X. He, "On Finding Disjoint Paths in Single and Dual Link Cost Networks," in Proc. INFOCOM, Hong Kong, March 2004.

[4] C.-L. Li, S.T. McCormick, and D. Simchi-Levi, "Finding disjoint paths with different path costs: Complexity and algorithms," in Networks, vol. 22, 1992, pp. 653-667.

[5] R. Andersen, F. Chung, A. Sen and G. Xue, "On disjoint path pairs with wavelength continuity constraint in WDM networks," IEEE INFOCOM 2004, pp. 524-535.

[6] J. Q. Hu, "Diverse Routing in Optical Mesh Networks", IEEE Transactions on Communications, vol. 51, No. 3, pp.489-494 2003.

[7] G. Li, R. Doverspike, and C. Kalmanek, "Fiber Span Failure Protection in Mesh Optical Networks", in OptiComm 2001: Optical Networking and Communications, Proc. SPIE, vol. 4599, pp. 130-142, 2001

[8] L. Shen, X. Yang and B. Ramamurthy, "Shared Risk Link Group (SRLG)-Diverse Path Provisioning under Hybrid Service Level Agreements in Wavelength-Routed Optical Mesh Networks: Formulation and Solution Approaches," SPIE OptiComm 2003, Dallas, TX, Oct. 2003

[9] D. Xu, Y. Xiong, C. Qiao, and G. Li, "Trap Avoidance and Protection Schemes in Networks with Shared Risk Link Groups", IEEE Journal of Lightwave Technology, vol. 21, no. 11, pp. 2683-2693, Nov. 2003.

[10] D. Xu, Y. Xiong and C. Qiao, "A New PROMISE Algorithm in Networks with Shared Risk Link Groups," IEEE Globecom, San Francisco, CA, Dec. 2003.

[11] P. Datta, A. K. Somani, "Diverse Routing for Shared Risk Resource Group (SRRG's) in WDM Optical Networks," in Proceedings of First Annual International Conference on Broadband Networking (BroadNets 04), San Jose, CA, pp. 120129 , Oct 2004

[12] A. Todimala and B. Ramamurthy, "IMSH: An Iterative Heuristic for SRLG-diverse Routing in WDM Mesh Networks," in Proceedings of IEEE Thirteenth International Conference on Computer Communications and Networks (ICCCN 2004), Chicago, IL, pp. 199-204, Oct 2004.

[13] P-H. Ho, J. Tapolcai, and H. T. Mouftah, "On Achieving Optimal Survivable Routing for Shared Protection in Survivable Next-Generation Internet," IEEE Transactions on Reliability (accepted).

[14] J. Y. Yen, "Finding the k shortest loop less paths in a network," Management Science vol. 17, pp. 712-716, 1971. 